\title{
COMMENTARY
}

\section{Prediction of fluid challenge effect: filling pressure when left ventricular function is abnormal, diastolic volume when left ventricular function is normal}

\author{
Didier Payen* \\ See related research by Trof et al., http://ccforum.com/content/15/1/R73
}

\begin{abstract}
Fluid resuscitation is a cornerstone of intensive care unit patient care, but prediction of the cardiovascular response remains difficult, despite many efforts in clinical research. The concept of responders and nonresponders illustrates such a difficulty. Many techniques have been tested, from strictly non-invasive to invasive, delivering various parameters related to the fluid challenge response. Considering the physical parameters available, such as pressure, volume and flow generated by right and left pumps circulating in elastic or compliant tubes, it sounds useful to go back to the basic knowledge to discuss the results of the present article. This published study tested in the postoperative period of cardiovascular surgery the prediction obtained with filling pressures and the diastolic volume. When left ventricular function (global ejection fraction) is adequate, the volume before fluid administration seems to predict well the fluid challenge response; whereas when the global ejection fraction is poor, the filling pressure seems more suitable. The present commentary discusses the main physiological issues related to these findings, with some methodological aspects.
\end{abstract}

When hypovolemia is strongly suspected, prediction of the fluid challenge response remains difficult for the intensivist or the anesthesiologist, because of complex interactions. In addition to the clinical art, each physician

*Correspondence: dpayen1234@orange.fr

Department of Anesthesiology \& Critical Care \& SAMU, Hôpital Lariboisière, Assistance Publique Hôpitaux de Paris \& University Paris 7, 2 rue Ambroise Paré, 75475 Paris Cedex 10, France wants to use numerical parameters to make the decision to give fluid and to ensure an adequate response to the fluid given. Apart from the debate on the type of fluid, the best way to evaluate the fluid challenge response has motivated a lot of clinical research aiming to demonstrate the specificity and sensitivity of several parameters, considering the invasiveness, the accuracy, and the cost of the methods.

In the past decade, many devices and parameters have been proposed to dynamically evaluate the response to fluid challenge. If fluid has to be given, the goal remains multifactorial, oscillating between hypotension correction, improvement in cardiac function, increase in cardiac output and oxygen delivery, or vascular recruitment. The effectiveness of this fluid challenge has been assessed by different methods: (1) echocardiography showing a better function of the right and left ventricles or better filled inferior or superior vena cava; (2) changes in oxygen delivery, which is simplified when arterial oxygen saturation is normal in changes in cardiac output, whatever the techniques for cardiac output measurements used; (3) changes in pulse pressure amplitude based on determinants of systolic and diastolic arterial blood pressure; or (4) cardiac filling pressure variations in association with cardiac output, according to the Frank-Starling law, but with invasive methods.

The study from Trof and colleagues examines the interest in measuring cardiac filling pressures or the diastolic cardiac volume index (global end-diastolic volume index) in cardiac or vascular surgery in the postoperative period, in the presence or absence of systolic left ventricular dysfunction [1]. The later parameter the global ejection fraction (GEF) was obtained from the ratio between the stroke volume and global end-diastolic volume (GEDV), being $>20 \%$ when left ventricular function is normal and $<20 \%$ when abnormal. These parameters have been obtained using the precursor technique of the pulse contour method (COLD Z-021; Pulsion 


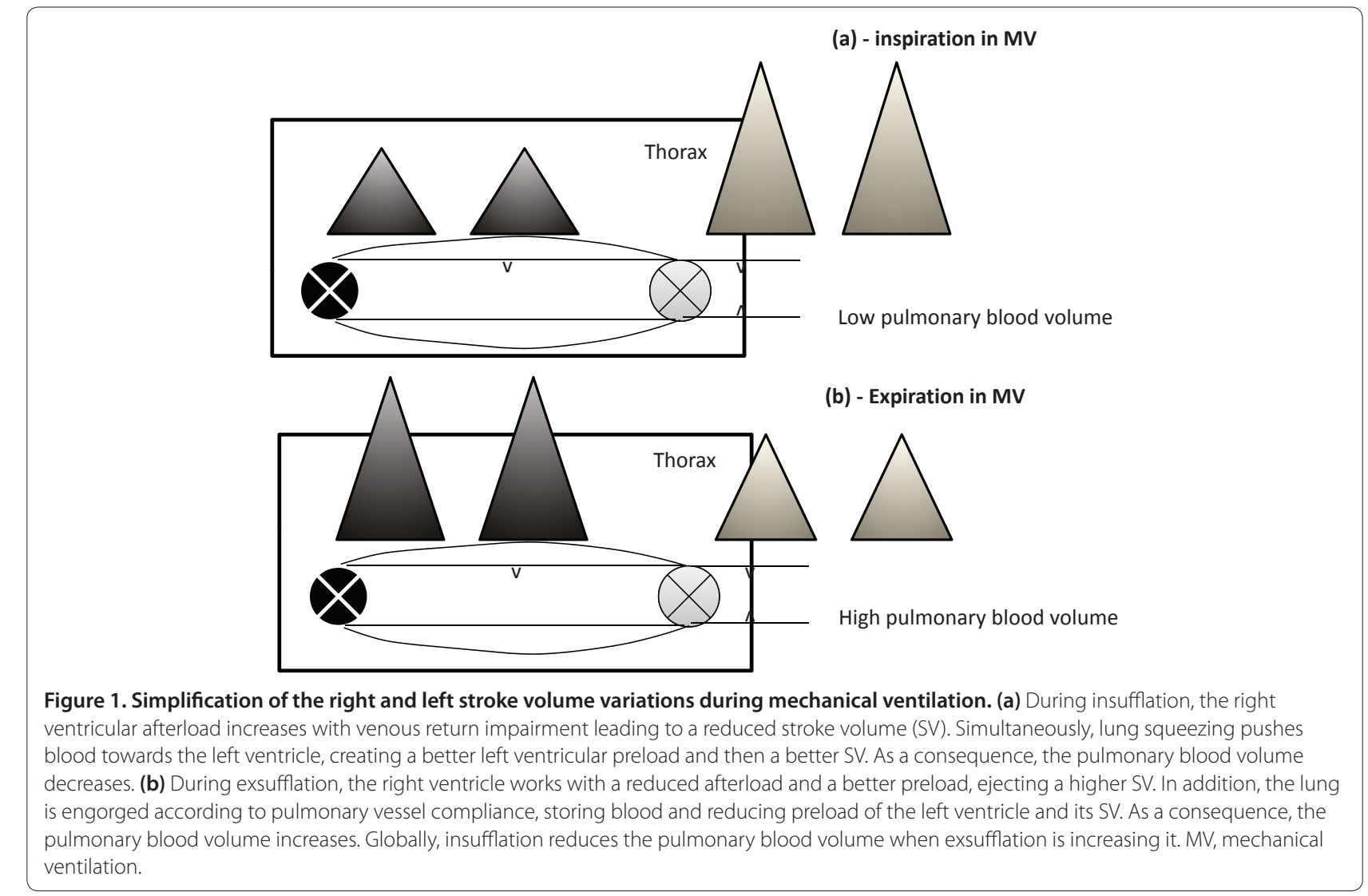

Medical Systems, Munich, Germany) with a double indicator. The carefully performed study from Trof and colleagues, with great caution for statistical analysis, concluded that regardless of the GEF the central venous pressure may be useful for predicting fluid responsiveness in these patients when the positive end-expiratory pressure (PEEP) is low. When the GEF is low $(<20 \%)$, the pulmonary artery occlusion pressure is more useful than the global end-diastolic volume index for predicting fluid responsiveness; but when the GEF is near normal $(\geq 20 \%)$, the global end-diastolic volume index is more useful than the pulmonary artery occlusion pressure.

Fluid responsiveness in terms of hemodynamic improvements deals with pressure, volume, cardiac pumps (right and left), vascular compliance and resistance, and circulatory time. Prediction for fluid responsiveness is difficult because of all these interactive factors, but some logical aspects can be taken on the basis of the results presented.

First, all of these conclusions are based on three major conditions: the presence of hypovolemia, a low PEEP level, and relatively adequate right ventricular function three restrictive conditions that are not frequently observed in intensive care unit patients. PEEP is frequently higher, as is the intrathoracic pressure - a situation that may alter the right ventricular function, with important modifications in pulmonary blood volume during the respiratory cycle (Figure 1). During inspiration on mechanical ventilation, the right ventricular afterload and reduced venous return both alter the right ventricular stroke volume towards the pulmonary vessels, which are compressed by intra-alveolar pressure. This lung squeezing as a sponge pushes the blood towards the left ventricle, being better filled and then ejecting a higher stroke volume. As a consequence, the pulmonary blood volume is reduced, with an inverse effect during expiration.

The amplitude of the variations between inspiration and expiration depends on the central blood volume, the pulmonary vascular capacitance, and pump function. The better the pulmonary vessel capacitance, the more the pulmonary vasculature may store blood, which will be pushed during mechanical ventilation insufflation to the left ventricle. This pulmonary blood volume is a part of the GEDV, which also includes the right and left heart volumes in diastole. The GEDV value comes from the product of cardiac output and the mean transit time for blood to flow from the right to the left side of the circulation (CO x MTT) [2]. If cardiac output can be considered adequately measured, the mean transit time might be more complex, since it is based on time determination for the geometric mean value of the 


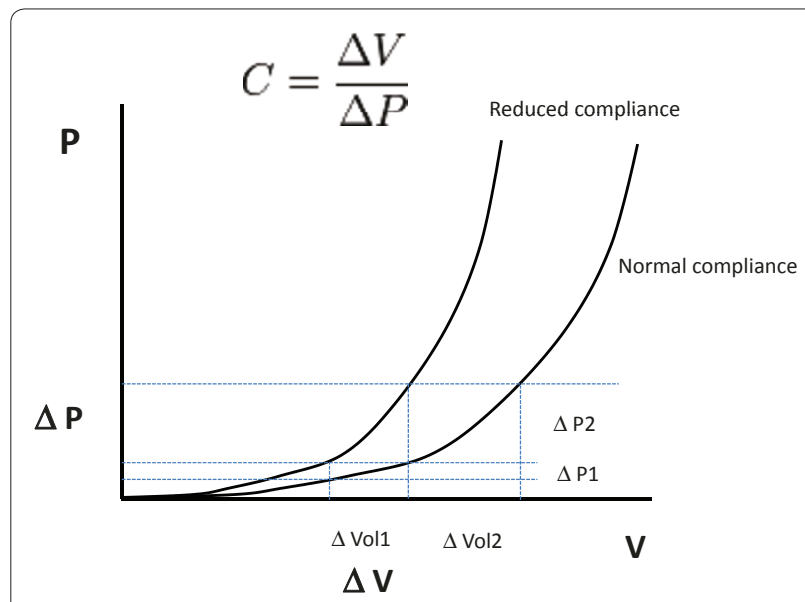

Figure 2. Ventricular compliance. When the change in volume/ pressure $(\Delta V / \Delta P)$ is normal, the increase in volume is larger than the increase in pressure when the preload is reduced during hypovolemia. Conversely, when compliance is reduced during hypovolemia, the increase in volume generates a larger change in pressure than in volume. P, pressure; $V$, volume.

intravascular indicator to flow (time of injection to the mean time), with potential errors occurring when ventricles have limited functions or valve regurgitations $[3,4]$.

Second, the conclusion from this study supposes that the right ventricle is pumping blood normally towards the left ventricle, which leads to a predominant role for left ventricular function on central blood volume size and on the filling pressure level. When systolic function is normal, therefore, the left ventricular volume after systole is small, suggesting that diastolic filling works on the flat part of the heart pressure/volume curve (Figure 2). As shown by the study, in this condition the filling volume would better predict fluid responsiveness since the volume is not limited (Figure 2) and pressure variations are small. When systolic function is limited, the end-systolic volume is higher with an end-diastolic volume located on the steep part of the pressure/volume curve (Figure 2). As a consequence, the filling pressure being higher, diastolic pressure better predicted the fluid responsiveness than did volume.

To conclude, the results from this nice study fit well with the pathophysiology when the PEEP is low, right ventricular function is adequate, and the patients are hypovolemic. These conditions are not typically those encountered in complex intensive care unit patients, and cannot be generalized. In addition, the preload index GEDV is more complex than the heart volumes in diastole, since it also contains the pulmonary blood volume, which may vary largely during mechanical ventilation and when pulmonary vascular compliance changes.

\section{Abbreviations}

GEDV, global end-diastolic volume; GEF, global ejection fraction; PEEP, positive end-expiratory pressure.

\section{Acknowledgements}

The present work was supported in part by a grant from University Paris 7 (Plan quadrennial, EA 3509).

\section{Competing interests}

The author declares that he has no competing interests.

\section{Published: 24 March 2011}

\section{References}

1. Trof RJ, Danad I, Wing-Lun Reilingh W, Breukers RM, Groeneveld ABJ: Cardiac filling volumes versus pressures for predicting fluid responsiveness after cardiovascular surgery: the role of systolic cardiac function. Crit Care 2011, 15:R73.

2. Bondurant S, Hickam JB: Circulatory and pulmonary effects of acute central vascular engorgement in normal subjects. Dis Chest 1959, 35:83-87.

3. Bigatello LM, Kistler EB, Noto A: Limitations of volumetric indices obtained by trans-thoracic thermodilution. Minerva Anestesio/ 2010, 76:945-949.

4. Payen DM, Brun-Buisson CJ, Carli PA, Huet Y, Leviel F, Cinotti L, Chiron B: Hemodynamic, gas exchange, and hormonal consequences of LBPP during PEEP ventilation. J App/ Physio/ 1987, 62:61-70.

doi:10.1186/cc10067

Cite this article as: Payen D: Prediction of fluid challenge effect: filling pressure when left ventricular function is abnormal, diastolic volume when left ventricular function is normal. Critical Care 2011, 15:139. 